\title{
Structural effect of poly(ethylene glycol) segmental length on biofouling and hemocompatibility
}

\author{
Chih-Chen Yeh $^{1}$, Antoine Venault ${ }^{1}$ and Yung Chang ${ }^{1,2}$
}

\begin{abstract}
Although numerous studies have focused on poly(ethylene glycol) methyl ether methacrylate (PEGMA) hydrogels, the effect of the molecular weight and crosslinking of the monomer in its nonfouling properties remains poorly understood. Here, we prepared a series of PEGMA hydrogels from monomers of various chain lengths and identified structure/property correlations. The results revealed that the hydrogel hydration capacity depended on the chain length and crosslinking (from X-ray photoelectron spectroscopy analysis): for a similar initial amount of crosslinker, a lower monomer molecular weight resulted in a higher degree of crosslinking and a lower level of hydration. Protein (fibrinogen, lysozyme, human and bovine serum albumin) adsorption and bacterial attachment tests indicated that the structure of the PEGMA 500 hydrogel was suitable (chain length, crosslinking) to minimize biofouling. A higher monomer molecular weight (as in PEGMA950) was associated with a higher hydrophilicity, but excessive free water in the cavities likely mediated foulant diffusion and trapping in the network. Conversely, a lower monomer molecular weight did not permit sufficient hydration to form a protective layer against fouling. These results were supported by blood cell (erythrocytes, leukocytes and thrombocytes) adhesion tests, suggesting that hemocompatibility can also be finely tuned by carefully selecting the monomer that constitutes the hydrogels.
\end{abstract}

Polymer Journal (2016) 48, 551-558; doi:10.1038/pj.2016.5; published online 24 February 2016

\section{INTRODUCTION}

Since Whitesides and colleagues ${ }^{1}$ established the criteria for nonfouling interfaces 15 years ago, the body of knowledge on the development of these interfaces has grown in importance. Briefly, a material should be hydrophilic, contain hydrogen-bond acceptors rather than donors and be electrically neutral to resist fouling. A number of materials exhibit these specific criteria and are currently classified into three major classes: poly(ethylene glycol) (PEG) derivatives, ${ }^{2-5}$ zwitterionic brushes $^{6-9}$ and mixed-charge moieties. ${ }^{10-12}$ In addition, the nonfouling nature of an interface is also believed to depend on other essential criteria of the brushes. In the case of surface modification and the growth of nonfouling moieties directly on a substrate, the ratio of hydrophobic to hydrophilic blocks (if a diblock/triblock copolymer is at play), the length of brushes, their architecture, their packing and their flexibility or orientation can also significantly affect the degree of fouling. ${ }^{13,14}$ Furthermore, the amount of crosslinks between the chains also affects fouling. Hence, Chang et al. ${ }^{15}$ showed that modifying the surfaces of poly(vinylidene fluoride) membranes by plasma treatment using poly(ethylene glycol) methyl ether methacrylate (PEGMA) as a surface modifier altered the organization of the PEGMA layer to result in free brushes or a network-like configuration. This modification depended on the type of plasma treatment applied (either atmospheric or low pressure). ${ }^{15}$ The organization of the polymer brushes significantly affected the extent of hydration, the nature of hydration and, consequently, the antifouling properties of the membrane. This effect also applies to polymer gels because the degree of crosslinking controls the extent of swelling of the gel, irrespective of the nature of crosslinks (ionic or covalent), and many properties depend on this swelling. ${ }^{16}$

PEG derivatives constitute the second generation of nonfouling materials because they were developed after hydroxyethylmethacrylate-based materials. Today, they are extensively used in various domains and under different physicochemical states. Hence, they can be used to modify dry, dense polymer films or porous membranes by coating, ${ }^{17,18}$ grafting $^{19}$ or in situ modification. ${ }^{20,21}$ They are also very popular as hydrogel matrices, either alone or combined with other polymers. ${ }^{22-30}$ They have been successfully applied in numerous fields, including water treatment, blood treatment, pharmaceutics or wound dressings. Despite some self-oxidation concerns that can alter the nonfouling properties in the long term, PEGylated antifouling matrices remain popular and have been widely investigated because they are readily prepared in a well-controlled manner. In addition, because these derivatives bear no charge, the $\mathrm{pH}$ of the environment in which they are used does not influence their nonfouling power, whereas mixed-charge brushes, for example, are subject to this influence.

Despite extensive research on this topic, some questions remain unanswered and some directions uninvestigated, especially the effect of the chain length (molecular weight) and crosslinks on the biofouling properties of PEGMA hydrogels. However, we believe that similar to

\footnotetext{
${ }^{1}$ Department of Chemical Engineering and R\&D Center for Membrane Technology, Chung Yuan Christian University, Chung-Li, Taiwan and ${ }^{2}$ Department of Botany and Microbiology, College of Science, King Saud University, Riyadh, Kingdom of Saudi Arabia

Correspondence: Professor Y Chang, Department of Chemical Engineering and R\&D Center for Membrane Technology, Chung Yuan Christian University, Chung-Li 32023, Taiwan.

E-mail: ychang@cycu.edu.tw

Received 28 September 2015; revised 23 December 2015; accepted 23 December 2015; published online 24 February 2016
} 
PEG-derivative surface-modified dry matrices, ${ }^{31}$ controlling this specific parameter when forming PEGMA hydrogels would further help to regulate the hydration properties of the gel and mitigate interactions with biofoulants, such as proteins, bacteria or other cells.

To meet these needs and find answers, we conducted a series of tests that are presented herein. We report the preparation of different PEGMA-analogous hydrogels with controlled network chain lengths. After chemical characterization, the hydration properties (surface and bulk) of the gels are presented. We then describe the influence of the chain length of the polymeric gels on the biofouling resistance at the nanoscale based on several protein adsorption tests. We also describe this relationship at the macroscale using bacteria and blood cells. The latter tests revealed the effect of the gel structure on its hemocompatibility.

\section{MATERIALS AND METHODS}

\section{Materials}

PEGMA homopolymers (number-average molecular weight $\left(M_{\mathrm{n}}\right)=$ $300 \mathrm{~g} \mathrm{~mol}^{-1}, \quad n=4.54 ; \quad M_{\mathrm{n}}=500 \mathrm{~g} \mathrm{~mol}^{-1}, \quad n=9.09 ; \quad M_{\mathrm{n}}=950 \mathrm{~g} \mathrm{~mol}^{-1}$, $n=9.50$ ), triethylene glycol methyl ether methacrylate (TEGMA, weightaverage molecular weight $\left.\left(M_{\mathrm{w}}\right)=232.27 \mathrm{~g} \mathrm{~mol}^{-1}\right)$ and $N, N, N^{\prime}, N^{\prime}$-tetramethylethylenediamine $\left(M_{\mathrm{w}}=116.2 \mathrm{~g} \mathrm{~mol}^{-1}\right)$ were purchased from Aldrich (Kaohsiung, Taiwan). $N, N^{\prime}$-methylenebis(acrylamide) $\left(M_{\mathrm{w}}=154.17 \mathrm{~g} \mathrm{~mol}^{-1}\right)$ and ammonium peroxodisulfate $\left(M_{\mathrm{w}}=218.2 \mathrm{~g} \mathrm{~mol}^{-1}\right)$ were purchased from Acros Organic (Thermo Fisher Scientific, Springfield Township, NJ, USA) and Showa Chemical (Tokyo, Japan), respectively. 2-Methoxyethyl acrylate (MEA, $M_{\mathrm{w}}=130.14 \mathrm{~g} \mathrm{~mol}^{-1}$ ) was purchased from Alfa Aesar (Ward Hill, MA, USA). Deionized water was obtained from a reverse-osmosis water-purification system (Millipore, Taipei, Taiwan) and presented a resistivity exceeding $18.0 \mathrm{M} \Omega \cdot \mathrm{cm}$. All proteins used in this work, bovine serum albumin (BSA), lysozyme (LY), fibrinogen (FN) and human serum albumin (HSA), were purchased from Sigma (Kaohsiung, Taiwan); phosphate-buffered saline (PBS) was also purchased from Sigma.

\section{Methods}

Preparation of hydrogels. The hydrogels were prepared according to the following protocol. First, a solution containing the monomer (MEA, TEGMA, $\mathrm{PEGMA}_{300}, \mathrm{PEGMA}_{500}$ or PEGMA 950$)$, water, crosslinker $\left(N, N^{\prime}\right.$-methylenebis (acrylamide) and catalyst ( $N, N, N^{\prime}, N^{\prime}$-tetramethylethylenediamine) was stirred for $30 \mathrm{~min}$. Then, initiator (ammonium peroxodisulfate) was added to the monomer solution. The molar ratio of the monomer, crosslinker, catalyst and initiator was 90:10:1:1, and the solid content was fixed at $20 \mathrm{wt} \%$. Once the initiator was added, the solution was immediately placed between glass slide plates that were placed in an oven maintained at $60^{\circ} \mathrm{C}$ for $1 \mathrm{~h}$ to start the reaction. After the reaction, the hydrogels were thoroughly washed with deionized water, followed by three washes with PBS to remove the unreacted monomer. The hydrogels were ultimately stored in a $10 \%$ ethanol solution in deionized water (volume $\%$ ). A schematic of the preparation is presented in Figure 1.

Physicochemical characterization of hydrogels. Hydrogels were characterized by X-ray photoelectron spectroscopy with a PHI Quantera SXM/Auger instrument (Chanhassen, MN, USA) working with a monochromated $\mathrm{Al} \mathrm{K} \mathrm{X-ray} \mathrm{source}$ (1486.6 eV photons). The following settings were used. A hemispherical energy analyzer (pass energy between 50 and $150 \mathrm{eV}$ ) was used to measure the energy of emitted electrons. A photoelectron take-off angle of $45^{\circ}$ (with respect to the gel surface) was set for all hydrogel analyses. In addition, the peak maximum in the $\mathrm{C} 1 \mathrm{~s}$ spectrum was set to $284.6 \mathrm{eV}$ that served as the binding energy scale reference. All data were analyzed using the Service Physics software (Bend, OR, USA).

The hydrophilicity of hydrogels was evaluated by measuring their hydration capacity (which relates to the extent of water molecules trapped within the brushes at the surface of the gels). The hydration capacity of the gels was measured in water and in PBS as follows. The gels (diameter: $1.7 \mathrm{~cm}$ ) were weighed and then immersed in water or PBS for $24 \mathrm{~h}$. Thereafter, the superficial water was gently wiped off, and the gels were weighed again. The difference per unit surface area between the dry and the wet weight of the gels was defined as the hydration capacity. Five measurements were conducted, and the average is reported in this work.

Protein adsorption tests. In this work, we tested the resistance to single commonly tested proteins: BSA $\left(\mathrm{MW} \approx 66000 \mathrm{~g} \mathrm{~mol}^{-1}\right)$ and LY

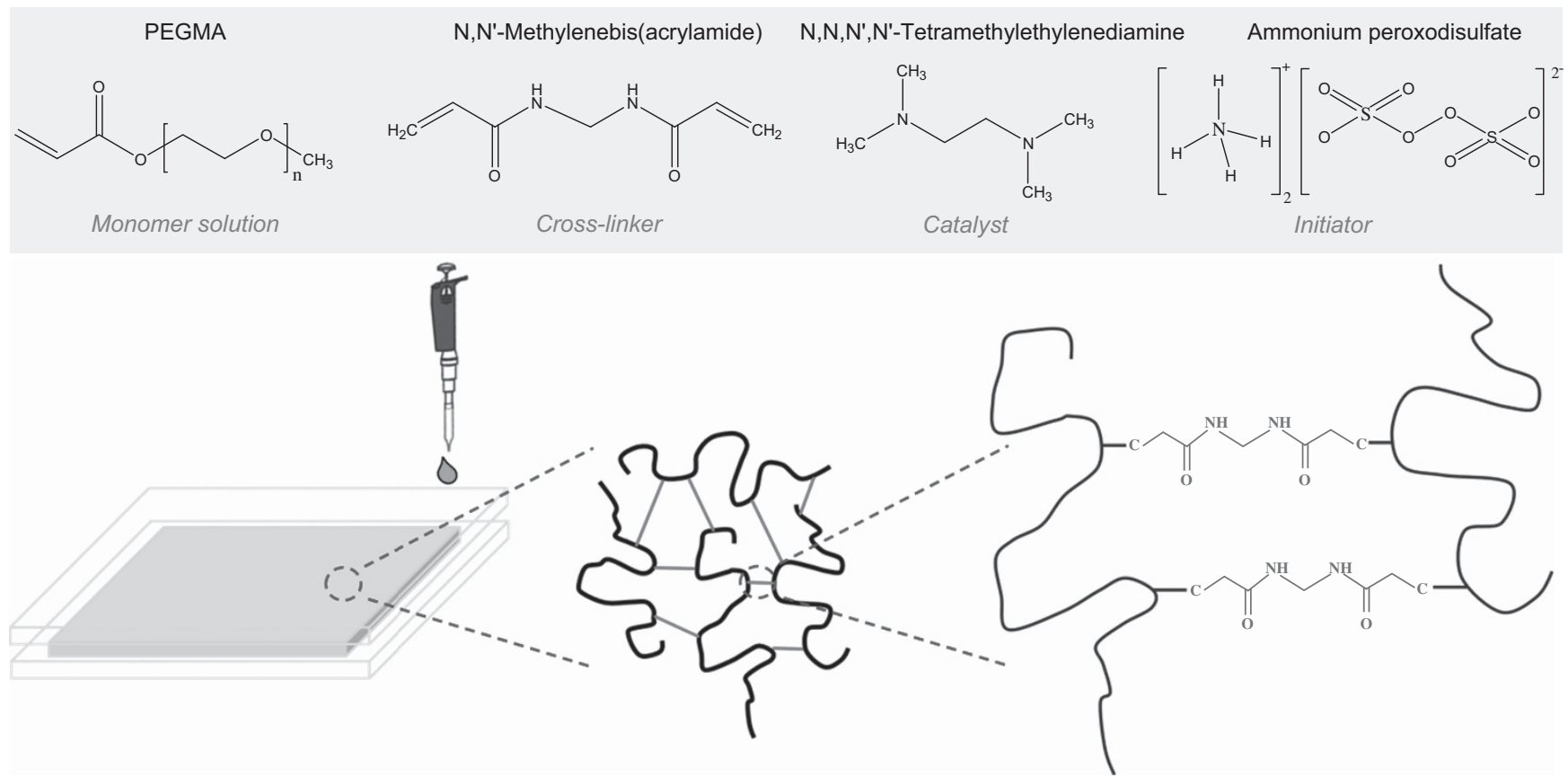

Figure 1 Schematic of the composition and preparation of hydrogels. PEGMA, poly(ethylene glycol) methyl ether methacrylate. A full color version of this figure is available at Polymer Journal online. 
$\left(\mathrm{MW}=14300 \mathrm{~g} \mathrm{~mol}^{-1}\right.$ ). The procedure for the adsorption tests of BSA and LY was the same and can be described as follows. The hydrogel disks (diameter: $1.7 \mathrm{~cm}$ ) were placed into individual wells of a 24-well plate and soaked in pure ethanol at ambient temperature $\left(\sim 25^{\circ} \mathrm{C}\right)$. After $30 \mathrm{~min}$, the ethanol was removed and replaced by PBS. The gels remained immersed in PBS for $2 \mathrm{~h}$. Subsequently, the gels were incubated at $25^{\circ} \mathrm{C}$ with either $1 \mathrm{ml}$ of BSA or $1 \mathrm{ml}$ of $\mathrm{LY}$ (the concentration in each case was $1 \mathrm{mg} \mathrm{ml}^{-1}$ ). To evaluate the extent of protein adsorbed by the gels, the absorbance of the protein solution after incubation was determined at $280 \mathrm{~nm}$ using a spectrophotometer (PowerWave XS, Biotech, Taoyuan, Taiwan (ROC)). Five independent tests were performed in each case, and the average is reported as the total amount of protein (BSA or LY) adsorbed by the gels.

We also tested the adsorption of proteins from a platelet-poor plasma solution: HSA (MW $\approx 67000 \mathrm{~g} \mathrm{~mol}^{-1}$ ) and FN (MW=340000 $\left.\mathrm{g} \mathrm{mol}^{-1}\right)$. For both, we performed an enzyme-linked immunosorbent assay test. The first step consisted of swelling the hydrogels that were initially placed in individual well of a 24-well plate to promote the diffusion of protein. Specifically, the gels were immersed in PBS for $1 \mathrm{~h}$ at $37^{\circ} \mathrm{C}$. Subsequently, the PBS was replaced by $1 \mathrm{ml}$ of a solution of FN or HSA in PBS $\left(1 \mathrm{mg} \mathrm{ml}^{-1}\right)$, and the gels were incubated with the protein solution at $37^{\circ} \mathrm{C}$ for $2 \mathrm{~h}$. The samples were then washed three times with PBS and incubated with a solution of BSA at $37^{\circ} \mathrm{C}$ for $1 \mathrm{~h}$ to block the sites unoccupied by the protein. The gel was again washed three times with PBS and subsequently immersed for $30 \mathrm{~min}$ at $37^{\circ} \mathrm{C}$ in $1 \mathrm{ml}$ of a solution of primary monoclonal antibody (US Biological, Salem, MA, USA). The hydrogels were then washed again three times with PBS before being incubated with secondary monoclonal antibody (horseradish peroxidase-conjugated, $1 \mathrm{ml}$ at $1 \mathrm{mg} \mathrm{ml}^{-1}$ ). Finally, PBS was used to rinse the samples five times, and the hydrogels were then transferred into clean individual wells. The final step consisted of incubating the gels with $0.5 \mathrm{ml}$ of a solution containing $1 \mathrm{mg} \mathrm{ml}^{-1}$ of 3,3',5,5'-tetramethylbenzidine chromogen, $0.05 \mathrm{wt} \%$ of hydrogen peroxide and $0.05 \mathrm{wt} \%$ of Tween- 20 at $37^{\circ} \mathrm{C}$ for $5 \mathrm{~min}$. The enzymatic reaction was then stopped using sulfuric acid $(500 \mu \mathrm{l})$, and the amount of adsorbed protein was determined by measuring the absorbance of the solution at $450 \mathrm{~nm}$ (with a PowerWave XS instrument, Biotech). The values reported in this study correspond to the average of five independent tests.

Bacterial attachment tests. Biofouling at the microscale was studied by carrying out bacterial attachment tests using Escherichia coli genetically modified with a green fluorescent protein, herein referred to as $E$. coli with green fluorescent protein (GFP). The genetic modification of $E$. coli is briefly explained as follows. Plasmid DNA pTrc-SFGFP $(2 \mu \mathrm{l})$ was added to $1.5 \mathrm{ml}$ tubes containing E. coli bacteria (BL21) and the tubes were placed on ice for $0.5 \mathrm{~h}$. The bacteria were then heat shocked by placing them in an oven maintained at $42{ }^{\circ} \mathrm{C}$ for $45 \mathrm{~s}$. LB medium $(0.5 \mathrm{ml})$ was added to the $1.5 \mathrm{ml}$ tube containing the cells, and the mixture was incubated and shaken for $1 \mathrm{~h}$ at $37^{\circ} \mathrm{C}$. Next, $0.1 \mathrm{ml}$ of the bacterial solution was deposited on an LB agar plate containing ampicillin $\left(50 \mu \mathrm{g} \mathrm{ml}^{-1}\right)$ to yield the modified bacteria $E$. coli with GFP. The bacteria were stored at $-20^{\circ} \mathrm{C}$ in $1.5 \mathrm{ml}$ tubes until use.

The next step consisted of culturing bacteria in an appropriate medium that contained the following: $3.0 \mathrm{mg} \mathrm{ml}^{-1}$ of beef extract and $5.0 \mathrm{mg} \mathrm{ml}^{-1}$ of peptone. This culture was continuously shaken at 100 r.p.m. and maintained at a temperature of $37^{\circ} \mathrm{C}$ until reaching the stationary phase that corresponded to a cell concentration of $10^{8}$ cells per $\mathrm{ml}$; this concentration was reached after $12 \mathrm{~h}$ of incubation. Then, $1 \mathrm{ml}$ of the bacterial suspension was incubated with the hydrogels at $37^{\circ} \mathrm{C}$ for $3 \mathrm{~h}$. The hydrogels were then washed three times with PBS and finally observed under a confocal microscope (NIKON CLSM A1R instrument, Tokyo, Japan). The images presented in this study were taken at $\lambda_{\mathrm{ex}}=488 \mathrm{~nm} / \lambda_{\mathrm{em}}=520 \mathrm{~nm}$. We focused only on live bacteria. The associated quantitative data were obtained by analyzing five independent samples for each condition.

Hemocompatibility tests. The hydrogels (diameter: $1.7 \mathrm{~cm}$ ) were placed in individual wells of a 24-well plate and equilibrated with $1 \mathrm{ml}$ of PBS at ambient temperature $\left(25^{\circ} \mathrm{C}\right)$. After $2 \mathrm{~h}$, the PBS was removed and replaced by $200 \mu \mathrm{l}$ of a solution of platelet-rich plasma prepared by centrifuging the fresh blood of a healthy volunteer at 1200 r.p.m. for $10 \mathrm{~min}$. The platelet-rich plasma was incubated with the hydrogels for $2 \mathrm{~h}$ at $37^{\circ} \mathrm{C}$. Then, the hydrogels were rinsed with PBS and immediately immersed overnight in a solution of glutaraldehyde $\left(2.5 \% \mathrm{v} / \mathrm{v}\right.$ in PBS) maintained at $4{ }^{\circ} \mathrm{C}$. Finally, the platelets adhering to the hydrogels were observed by confocal microscopy using the same instrument and settings as those used to study bacterial attachment.

The adhesion of red or white blood cells was assessed in a similar manner. After preparing red or white blood cell concentrate by centrifuging fresh blood at 1200 r.p.m. for $10 \mathrm{~min}$, the hydrogels (diameter: $1.7 \mathrm{~cm}$ ) were incubated with $200 \mu \mathrm{l}$ of this concentrate at $37^{\circ} \mathrm{C}$ for $2 \mathrm{~h}$. The samples were thoroughly rinsed with PBS and fixed with $300 \mu$ l of a solution of glutaraldehyde $(2.5 \%, \mathrm{v} / \mathrm{v}$ in PBS $)$ at $4{ }^{\circ} \mathrm{C}$ for $10 \mathrm{~h}$. The gels were again washed several times with PBS and observed by confocal microscopy in a manner similar to that used to observe platelets.

The protocol for the whole blood adhesion test is also very similar to the aforementioned protocols. After equilibrating the hydrogels (diameter: $1.7 \mathrm{~cm}$ ) with PBS in individual wells of a 24-well plate, $1 \mathrm{ml}$ of fresh blood was poured onto the hydrogels. Notably, the fresh blood $(250 \mathrm{ml})$ had been previously mixed with citrate phosphate dextrose adenine- $1(35 \mathrm{ml})$. The hydrogels were incubated in blood for $2 \mathrm{~h}$ at $37^{\circ} \mathrm{C}$. The hydrogels were then washed and immersed in a solution of formaldehyde $\left(4 \% \mathrm{v} / \mathrm{v}\right.$ in PBS) maintained at $4{ }^{\circ} \mathrm{C}$ for $15 \mathrm{~min}$. Finally, the hydrogels were rinsed with PBS, stained for $15 \mathrm{~min}$ at $4{ }^{\circ} \mathrm{C}$ with a solution of CD3-FITC, CD14-FTIC and CD45-FITC in PBS and observed by confocal microscopy, similar to the procedure described for the blood cell adhesion tests. The quantitative data for the blood cell adhesion tests were obtained by analyzing five independent samples for each condition.

\section{RESULTS}

\section{Physicochemical characterization of hydrogels}

The surface chemistry of hydrogels was characterized by X-ray photoelectron spectroscopy, and the related results are shown in Table 1 and Figure 2. The proportions of carbon and oxygen atoms were similar for the three PEGMA hydrogels ( $\sim 67$ At $\%$ of carbon and 29 At $\%$ of oxygen). However, the nitrogen content tended to decrease from MEA to TEGMA and inversely correlated with the molecular weight of PEGMA homopolymer, suggesting that the number of crosslinks in hydrogels decreased in the following order: MEA $<$ TEGMA $<$ PEGMA $_{300}<$ PEGMA $_{500}<$ PEGMA $_{950}$, where ' $<$ ' denotes for 'less crosslinked'. This relationship is also supported by quantifying the crosslinking ratio based on the core-level spectra of $\mathrm{N} 1 \mathrm{~s}, \mathrm{C} 1 \mathrm{~s}$ and $\mathrm{O} 1 \mathrm{~s}$ (Table 1). Indeed, the crosslinker, N,N'-methylenebis(acrylamide) introduced nitrogen atoms provided that the gels were well rinsed such that no catalyst $\left(N, N, N^{\prime}, N^{\prime}\right.$-tetramethylethylenediamine) remained. Thus, the molecular weight of hydrophilic moieties forming the hydrogel inversely correlated with the degree of crosslinking between the chains because the initial amount of crosslinker in the solutions before gelation was constant. Thus, there is a larger free volume between the chains forming the polymeric network when moving from MEA to $\mathrm{PEGMA}_{950}$.

Table 1 Atomic content of hydrogels determined based on the XPS analysis

\begin{tabular}{lllllll}
\hline Sample ID & $C$ (At\%) & $N$ (At\%) & O (At\%) & $S$ (At\%) & $\begin{array}{c}\text { Others } \\
(\text { At\%) }\end{array}$ & $\begin{array}{c}N 1 s /(C 1 s+01 s \\
+N 1 s)\end{array}$ \\
\hline TCPS & 88.56 & 0 & 11.44 & 0 & 0 & 0 \\
MEA & 50.42 & 4.10 & 43.80 & 1.24 & 0.44 & 0.044 \\
TEGMA & 56.27 & 3.24 & 38.64 & 1.68 & 0.17 & 0.033 \\
PEGMA $_{300}$ & 66.66 & 2.66 & 28.75 & 1.22 & 0.71 & 0.027 \\
PEGMA $_{500}$ & 66.49 & 2.31 & 29.36 & 0.74 & 1.10 & 0.023 \\
PEGMA $_{950}$ & 67.22 & 1.96 & 29.02 & 0.66 & 1.14 & 0.020 \\
\hline
\end{tabular}

Abbreviations: MEA, 2-methoxyethyl acrylate; PEGMA, poly(ethylene glycol) methyl ether methacrylate; TCPS, tissue culture polystyrene; TEGMA, triethylene glycol methyl ether methacrylate; XPS, X-ray photoelectron spectroscopy. 


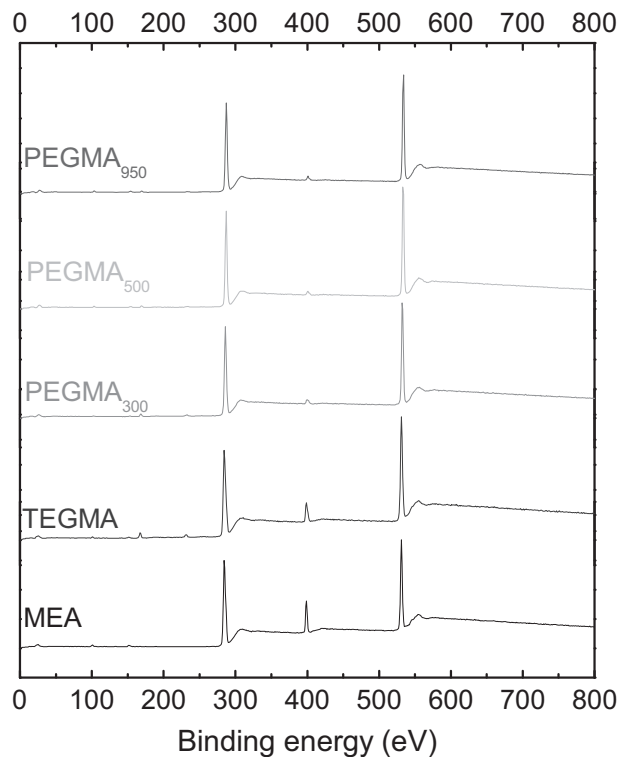

Figure 2 XPS analysis of the different hydrogels. MEA, 2-methoxyethyl acrylate; PEGMA, poly(ethylene glycol) methyl ether methacrylate; TEGMA, triethylene glycol methyl ether methacrylate; XPS, X-ray photoelectron spectroscopy. A full color version of this figure is available at Polymer Journal online.

The hydrophilicity of hydrogels was evaluated by measuring their hydration capacity that reflects the overall hydrophilicity of hydrogels and the ability of PEGMA chains to trap water molecules. The hydration capacity is provided in $\mathrm{mg}$ of water trapped per unit surface area, but measuring the hydration capability, which is a volume-based measurement (in $\mathrm{mg} \mathrm{cm}^{-3}$ ), would have provided the same results because the thickness of the gels was constant. In addition, the hydration capacity was evaluated in water or PBS. The results shown in Figure 3 reveal that among the PEGMA series, PEGMA $_{950}$ gels exhibited the highest global surface (or bulk) hydrophilicity, whereas $\mathrm{PEGMA}_{300}$ gels exhibited the poorest hydrophilicity properties. Consequently, the hydrophilic properties of PEGMA 500 fall between these two extremes. In addition, the hydration capacities of the MEA and TEGMA gels were the lowest, which is attributed to the low molecular weight and high degree of crosslinking, that hindered the formation of $\mathrm{H}$-bonds with water and the diffusion of water molecules through the network, respectively. Moreover, the medium affected the hydration capacity. For instance, the hydration capacity of PEGMA 300 gels was $3.68 \pm 0.03 \mathrm{mg} \mathrm{cm}^{-2}$ in water and $3.24 \pm 0.04 \mathrm{mg} \mathrm{cm}^{-2}$ in PBS. This difference arises because the ions in PBS and outside the gel matrix are solvated by free water molecules. Thus, these molecules are not available to diffuse in the gels and contribute to their swelling.

\section{Resistance of hydrogels to protein adsorption}

Nano-biofouling is a major concern in numerous applications of materials and is commonly studied by carrying out protein adsorption tests. $^{31-33}$ In this work, we used BSA, LY, fibrinogen and HSA as model proteins to study fouling by wastewaters or biological fluids (such as blood plasma). In addition, we used several proteins of various molecular weights and molecular structures to provide a more realistic picture of the actual biofouling resistance of the prepared hydrogels. The results displayed in Figure 4 reveal that PEGMA hydrogels all resist protein adsorption very well, better than MEA and TEGMA gels. Furthermore, the PEGMA 500 hydrogel, an intermediate

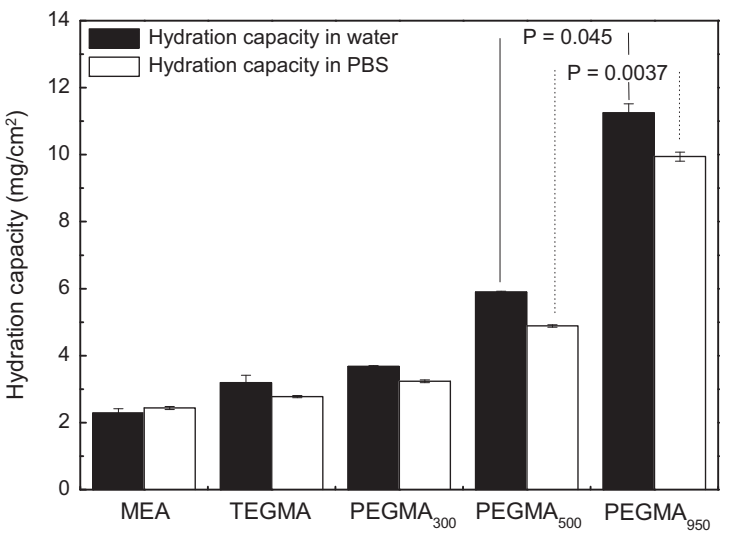

Figure 3 Hydration properties of hydrogels. MEA, 2-methoxyethyl acrylate; PBS, phosphate-buffered saline; PEGMA, poly(ethylene glycol) methyl ether methacrylate; TEGMA, triethylene glycol methyl ether methacrylate.
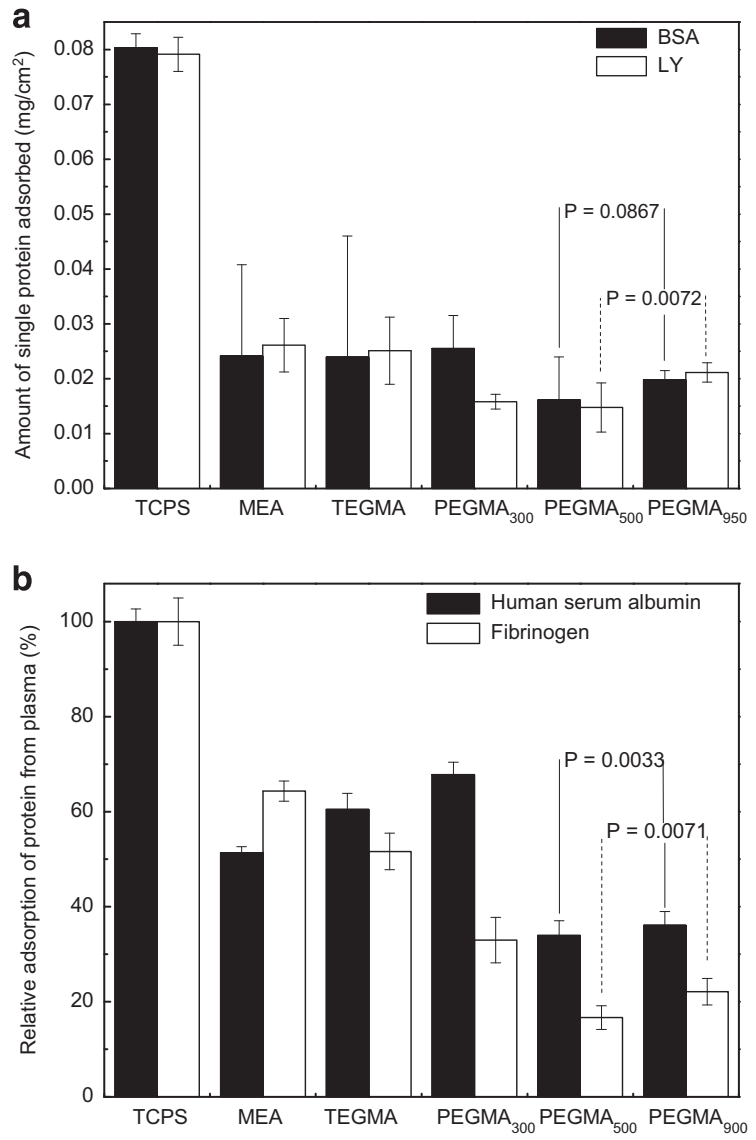

Figure 4 Resistance of hydrogels to the adsorption of (a) model single protein from water and (b) proteins from blood plasma. BSA, bovine serum albumin; LY, lysozyme; MEA, 2-methoxyethyl acrylate; PEGMA, poly(ethylene glycol) methyl ether methacrylate; TCPS, tissue culture polystyrene; TEGMA, triethylene glycol methyl ether methacrylate.

molecular weight hydrogel, offers the best biofouling resistance for all proteins tested. Hence, the final levels of BSA and LY for these gels were $0.0161 \pm 0.008$ and $0.0148 \pm 0.005 \mathrm{mg} \mathrm{cm}^{-2}$, respectively. PEGMA $_{500}$ hydrogel was also most resistant to fouling from proteins from a platelet-poor plasma solution, that is, FN and HSA, with final relative adsorptions of $16.7 \pm 2.5 \%$ and $34.0 \pm 3.1 \%$, respectively, 
relative to the positive control (tissue culture polystyrene that was defined as $100 \%$ adsorption).

\section{Resistance of hydrogels to bacterial attachment}

To better understand the resistance of hydrogels to biofouling at a different scale, we performed bacterial attachment tests, and the related qualitative and quantitative results are shown in Figures 5 and 6, respectively. Our confocal analysis revealed that numerous bacteria adhered to the control tissue culture polystyrene plate and the control MEA and TEGMA hydrogels. The nonfouling nature of PEGMA hydrogels was evident in all images, but PEGMA ${ }_{500}$ and PEGMA $_{950}$ gels appeared to resist bacterial attachment better than PEGMA $_{300}$ gels. This confocal analysis was confirmed by the quantitative analysis. Specifically, the cell densities on the hydrogel surface were $98 \pm 5,21 \pm 2$ and $24 \pm 3$ cells per $\mathrm{cm}^{2}$ for $\mathrm{PEGMA}_{300}$ hydrogel, PEGMA ${ }_{500}$ hydrogel and PEGMA 950 hydrogel, respectively. Therefore, PEGMA $_{500}$ hydrogel offers the best resistance to biofouling at the microscale.

\section{Effect of the chain length of PEGMA hydrogels on their hemocompatibility}

We then tested the hemocompatibility of hydrogels using blood cell attachment tests. These tests also reflect the resistance of hydrogels to micro-biofouling. We either employed single cells (erythrocytes, leukocytes or thrombocytes) or whole blood. Figures 7 and 8 present the confocal images and the related quantitative analysis, respectively, for both single blood cell attachment tests and whole blood attachment test. All PEGMA hydrogels were very resistant to the adhesion of blood cells, unlike the control tissue culture polystyrene or MEA and TEGMA hydrogels. In addition, PEGMA 500 gels again offer the best resistance to the adhesion of blood cells, with cell densities of $3 \pm 1, \quad 10 \pm 1, \quad 30 \pm 2$ and $28 \pm 2$ cells per $\mathrm{cm}^{2}$ for erythrocytes, leukocytes, thrombocytes and whole blood, respectively. The confocal images clearly show that all gels were hemocompatible compared with the controls. This behavior was expected based on previously published related works that demonstrated the hemocompatibility of PEG or PEG-based materials, ${ }^{34-36}$ and because a hemocompatible material is nonfouling for a specific species (it resists proteins from blood and blood cell adhesion). However, these results also suggest that the extent of hemocompatibility can also be controlled by the gel structure (chain length and crosslinking) and not only by the chemical composition of the hydrogel.

\section{DISCUSSION}

The goal of the present work was to analyze the antifouling properties of PEGMA analogs, that is, hydrogels prepared from different homopolymers. The results revealed that the molecular weight of the PEGMA homopolymer directly correlated with the overall hydrophilicity of the gel, which significantly influences fouling, unlike the local surface hydrophilicity, which is measured based on the

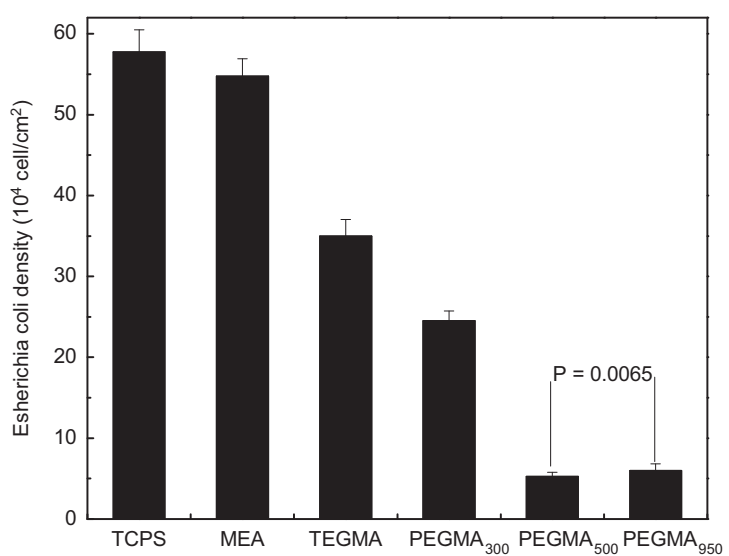

Figure 6 Quantitative analysis of the attachment of Escherichia coli to hydrogels. MEA, 2-methoxyethyl acrylate; PEGMA, poly(ethylene glycol) methyl ether methacrylate; TCPS, tissue culture polystyrene; TEGMA, triethylene glycol methyl ether methacrylate.
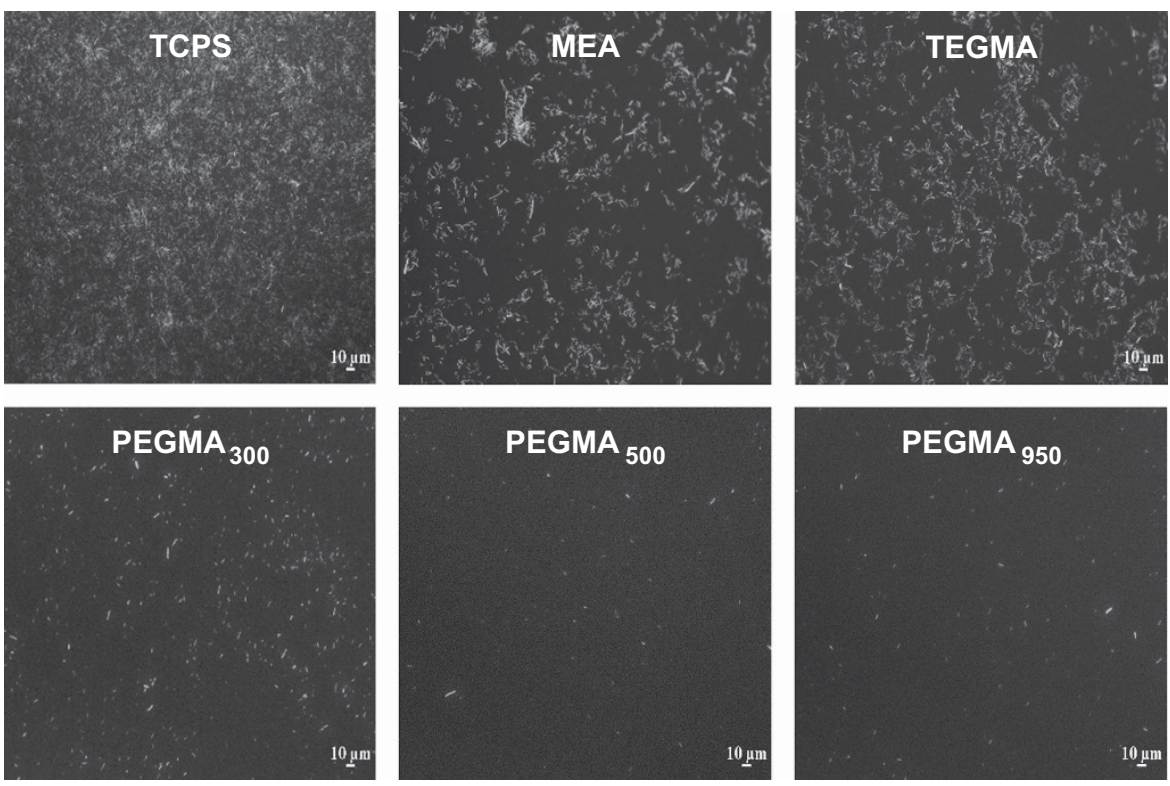

Scale bar: $30 \mu m$ -

Figure 5 Confocal images of the attachment of Escherichia coli to hydrogels. MEA, 2-methoxyethyl acrylate; PEGMA, poly(ethylene glycol) methyl ether methacrylate; TCPS, tissue culture polystyrene; TEGMA, triethylene glycol methyl ether methacrylate. A full color version of this figure is available at Polymer Journal online. 


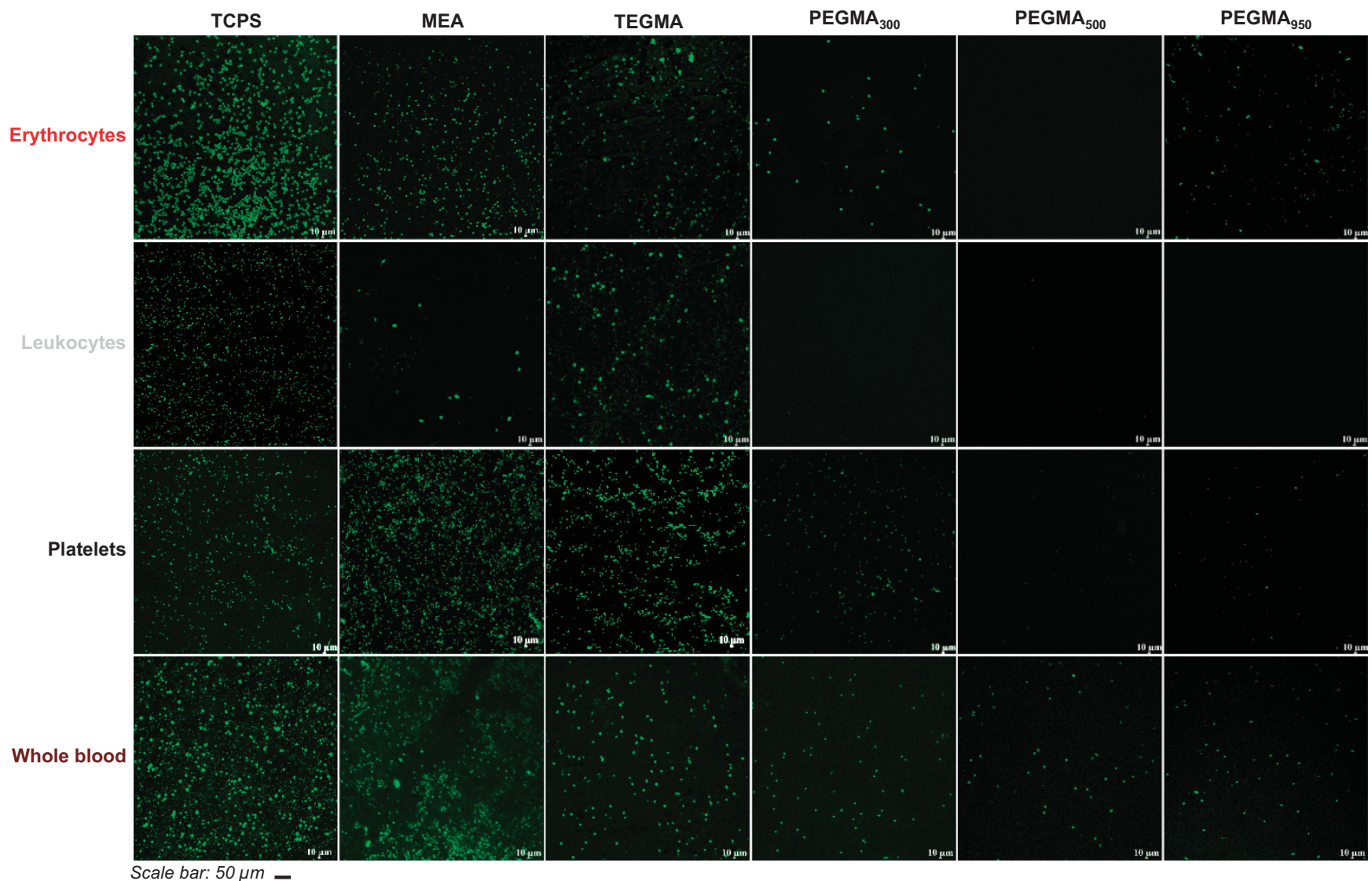

Figure 7 Confocal images of the attachment of blood cells to hydrogels. MEA, 2-methoxyethyl acrylate; PEGMA, poly(ethylene glycol) methyl ether methacrylate; TCPS, tissue culture polystyrene; TEGMA, triethylene glycol methyl ether methacrylate.

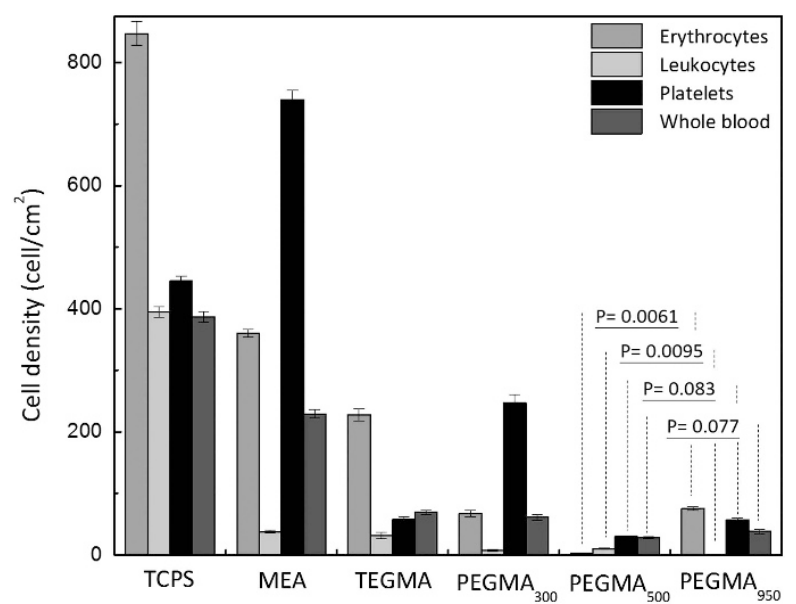

Figure 8 Quantitative analysis of the attachment of blood cells to hydrogels. MEA, 2-methoxyethyl acrylate; PEGMA, poly(ethylene glycol) methyl ether methacrylate; TCPS, tissue culture polystyrene; TEGMA, triethylene glycol methyl ether methacrylate. A full color version of this figure is available at Polymer Journal online.

contact angle. This observation likely arises from different crosslinking ratios between hydrogels. Although we lacked accurate data on the extent of crosslinking within the gels, an elemental analysis revealed that the homopolymer molecular weight directly correlated with the number of ethylene glycol repeat units and inversely correlated with the nitrogen content, that is, the proportion of crosslinkers in the gel structure decreased. Therefore, the free volume between polymeric chains of PEGMA 950 gels increases to facilitate water trapping within the chains and the bulk (higher water hydration capacity). Nonetheless, the high hydrophilicity of hydrogels is not associated with the optimized nonfouling properties, and PEGMA ${ }_{950}$ hydrogels consequently did not offer best resistance to the different proteins, bacteria and other cells.

The tests of the resistance to nano-biofouling revealed that PEGMA $_{500}$ hydrogels offered the best protection from the adhesion of proteins. These results were further supported by the observed biofouling by bacteria, that is, larger-scale biofouling tests. PEGMA 500 gels exhibit an intermediate hydration level, as discussed above. Gels presenting lower $\left(\mathrm{PEGMA}_{300}\right)$ and higher $\left(\mathrm{PEGMA}_{950}\right)$ hydration levels, although also offering good resistance to proteins, do not offer a protection as good as that of PEGMA $\mathrm{P}_{500}$ hydrogels. This observation may arise from the quality of the hydration. PEGMA ${ }_{300}$ gels are slightly less hydrophilic than PEGMA P00 $_{50}$ gels. Because the hydrophilicity correlates with the fouling resistance, PEGMA 500 gels likely better resist the adhesion of proteins than PEGMA $_{300}$ gels. However, PEGMA 950 gels are more hydrophilic than PEGMA $_{500}$ gels and should consequently offer even better protection against biofouling by proteins. However, we hypothesize that not all water molecules interact with the PEGylated chains in PEGMA ${ }_{950}$ gels. Instead, these gels exhibit more free water at the surface between the chains and within the structure because polymeric chains are longer and the extent of crosslinking is lower. This structure may affect nano-biofouling as follows: proteins diffuse more easily through 
the pores of the hydrogels using free water and then more readily reach potential interaction sites. A similar explanation applies to micro-biofouling: if the hydrophilicity is not sufficiently high, cells stick to the gels via their cell wall, whereas they can penetrate the polymeric chains more easily and become trapped within the hydrogel if the spaces between the hydrophilic moieties are too large. Controlling the structure of the hydrogel by controlling the length of the antifouling moieties and the extent of crosslinking between the gels appears to be extremely important to control the final hydration level, the type of hydration and, ultimately, the extent of nano- and micro-biofouling. However, the window to optimize hydration and minimize fouling is quite narrow: TEGMA and PEGMA ${ }_{500}$ contain 3 and 4.54 ethylene glycol units, respectively, whereas the molecular weight of TEGMA is $232.27 \mathrm{~g} \mathrm{~mol}^{-1}$ and that of PEGMA 500 is $300 \mathrm{~g} \mathrm{~mol}^{-1}$. Therefore, the hydration capacity of PEGMA ${ }_{500}$ is slightly higher than that of TEGMA (Figure 3), but the fouling significantly differed between these materials, suggesting that the molecular weight needs to be fine tuned for PEGMA analogs to control fouling.

The results of bacterial attachment were further supported by the observed blood cell attachment that also indicates micro-biofouling. Blood cells are deformable, and their size is on the same order of magnitude of that of E. coli bacteria. Thus, these results were expected. The observed FN adsorption also suggested that these hydrogels are resistant to blood cell fouling, especially platelet fouling. FN is known to mediate platelet adhesion and activation ${ }^{37,38}$ such that the hydrogel presenting the highest resistance to FN, PEGMA 500 was also expected to show improved resistance to the adhesion of platelets. Actually, the important information concerns again the fact that to better tune the hemocompatibility of a surface potentially coated by PEGMA moieties, one should carefully control first the length of the hydrophilic layer and the crosslinking to prevent facilitated trapping of blood cells within the network or hydrophobic interactions between the cell wall and the polymer. Blood cells will be trapped if the free volume between polymer chains is too large, whereas hydrophobic interactions can readily be established if too few water molecules constitute the hydrated protective layer around PEGMA moieties. The control of the spaces between polymer chains in the network is important, and blood cells are extremely deformable and can squeeze through capillaries significantly smaller than their diameter. In addition, they are very sensitive, and red blood cell lysis or platelet activation can readily occur if interactions with the polymeric network are established. Nonetheless, the size of the cavities, though essential in the control of fouling, is not the only parameter to take into account. Specific matrix/cell wall interactions also play a major role, as evidenced by the adsorption of leukocytes. Specifically, PEGMA 900 performed the best, suggesting the need for further investigations.

In conclusion, $\mathrm{PEGMA}_{500}$ gels offer the best compromise between hydrophilicity and physical structure, eventually allowing nanoscale biofouling by proteins and microscale fouling by bacteria or blood cells to be minimized. The growth of a PEGMA hydrogel on a material is already known as a good approach to avoid fouling, particularly in devices that contact blood, but this strategy can be further improved by controlling the chain length and the extent of crosslinking.

\section{CONCLUSION}

This work presented the preparation of various PEGMA-analogous hydrogels of a controlled polymer chain length and different degrees of crosslinking (measured based on the nitrogen content in the hydrogel) and how the structure of these hydrogels affected their nonfouling behavior. The following major conclusions were drawn:
- PEGMA $_{500}$ presented an intermediate overall hydrophilicity (hydration capacity) compared with MEA, TEGMA, PEGMA 300 (less hydrophilic) and PEGMA $_{950}$ (more hydrophilic, larger cavities), and this is attributed to both the intermediate hydrophilic chain length and intermediate crosslinking degree.

- The quality of hydration directly depends on the molecular weight and the crosslinking degree of hydrogels. Specifically, the chain length of hydrogels directly correlates with the hydration level. If the chain length of polymeric structure is high, the interactions with water molecules become weak and large amounts of free water circulate within the network, eventually mediating foulant diffusion in the structure toward the potential interaction sites.

- Nano-biofouling by single proteins commonly found in water and by plasma proteins was more efficiently mitigated on PEGMA P $_{500}$ hydrogels, indicating a strong correlation among the structure of the hydrogels, hydration quality and resistance to nano-biofouling.

- Micro-biofouling by bacteria and blood cells supported the conclusions drawn from the nano-biofouling experiments, that is, PEGMA $_{500}$ hydrogels generally offered the best resistance to E. coli modified with GFP, erythrocytes, platelets and whole blood.

Overall, the results of this work demonstrate that the structure and arrangement of polymer chains within a nonfouling system significantly affect resistance to fouling. The resistance of PEGMA hydrogels to fouling by biological materials, which has been widely studied in the literature, can be further tuned, and the perfect nonfouling PEGMA hydrogel has a wide range of potential applications, including the surface modification of membranes for water treatment, the coating of devices in contact with blood, the formation of wound dressings or the in vivo controlled release of drugs from nonfouling hydrogels. However, the pore size needs to be measured in future studies to verify assumptions based on chemical characterization and the effect on biofouling. In addition, the interactions between the cell walls of various cells and the hydrogel need to be studied to discriminate whether the structure or the chemical composition of the cells (for example, spectrin protein of erythrocytes, glycoproteins of thrombocytes and so on) primarily affects the fouling of these networks.

\section{CONFLICT OF INTEREST}

The authors declare no conflict of interest.

\section{ACKNOWLEDGEMENTS}

We acknowledge the Outstanding Professor Research Program at the Chung Yuan Christian University of Taiwan and the Ministry of Science and Technology (MOST 103-2221-E-033-078-MY3) for their financial support. The Deanship of Scientific Research at the College of Science Research Centre of King Saud University, Kingdom of Saudi Arabia, also supported this work.

1 Chapman, R. G., Ostuni, E., Takayama, S., Holmlin, R. E., Yan, L. \& Whitesides, G. M. Surveying for surfaces that resist the adsorption of proteins. J. Am. Chem. Soc. 122, 8303-8304 (2000)

2 Alcantar, N. A., Aydil, E. S. \& Israelachvili, J. N. Polyethylene glycol-coated biocompatible surfaces. J. Biomed. Mater. Res. 51, 343-351 (2000).

3 Guidpati, S. C., Finlay, J. A., Callow, J. A., Callow, M. E. \& Wooley, K. L. The antifouling and fouling-release performance of hyperbranched fluoropolymer (HBFP)-poly (ethyleneglycol) (PEG) composite coatings evaluated by adsorption of biomacromolecules and the green fouling alga Ulva. Langmuir 21, 3044-3053 (2005).

4 Zhao, W., Su, Y., Li, C., Shi, Q., Ning, X. \& Jiang, Z. Fabrication of antifouling polyethersulfone ultrafiltration membranes using Pluronic F127 as both surface modifier and pore-forming agent. J. Membr. Sci. 318, 405-412 (2008). 
5 Liu, B., Chen, C., Zhang, W., Crittenden, J. \& Chen, Y. Low-cost antifouling PVC ultrafiltration membrane fabrication with Pluronic $F$ 127: Effect of additives on properties and performance. Desalination 307, 26-33 (2012).

6 Yi, Z., Zhu, L. P., Xu, Y. Y., Gong, X. N. \& Zhu, B. K. Surface zwitterionicalization of poly(vinylidene fluoride) porous membranes by post-reaction of the amphiphilic precursor. J. Membr. Sci. 385-386, 57-66 (2011).

7 Li, M. L., Li, J. H., Shao, X. S., Ming, J. J., Wang, B., Zhang, Q. Q. \& Xu, X. P. Grafting zwitterionic brush on the surface of PVDF membrane using physisorbed free radical grafting technique. J. Membr. Sci. 405-406, 141-148 (2012).

8 Zhang, Z., Chao, T., Chen, S. \& Jiang, S. Superlow fouling sulfobetaine and carboxybetaine polymers on glass slides. Langmuir 22, 10072-10077 (2006).

9 Carr, L., Cheng, G., Xue, H. \& Jiang, S. Engineering the polymer backbone to strengthen nonfouling sulfobetaine hydrogels. Langmuir 26, 14793-14798 (2010).

10 Chen, S., Yu, F., Yu, Q., He, Y. \& Jiang, S. Strong resistance of a thin crystalline layer of balanced charged groups to protein adsorption. Langmuir 22, 8186-8191 (2006).

11 Chen, S. \& Jiang, S. A new avenue to nonfouling materials. Adv. Mater. 20, 335-338 (2008)

$12 \mathrm{Mi}$, L., Bernards, M. T., Cheng, G., Yu, Q. \& Jiang, S. pH responsive properties of nonfouling mixed-charge polymer brushes based on quaternary amine and carboxylic acid monomers. Biomaterials 31, 2919-2925 (2010).

13 Chang, Y., Chu, W. L., Chen, W. Y., Zheng, J., Liu, L., Ruaan, R. C. \& Higuchi, A. A systematic SPR study of human plasma protein adsorption behavior on the controlled surface packing of self-assembled poly(ethylene oxide) triblock copolymer surfaces. J. Biomed. Mater. Res. Part A 93, 400-408 (2010),

14 Gunkel, G., Weinhart, M., Becherer, T., Haag, R. \& Huck, W. T. S. Effect of polymer brush architecture on antibiofouling properties. Biomacromolecules 12, 4169-4172 (2001)

15 Chang, Y., Shih, Y. J., Ko, C. Y., Jhong, J. F., Liu, Y. L. \& Wei, T. C. Hemocompatibility of poly(vinylidene fluoride) membrane grafted with network-like and brush-like antifouling layer controlled via plasma-induced surface PEGylation. Langmuir 27, 5445-5455 (2011)

16 Feng, X. \& Pelton, R. Carboxymethyl cellulose: polyvinylamine complex hydrogel swelling. Macromolecules 40, 1624-1630 (2007).

17 Ding, X., Yang, C., Lim, T. P., Hsu, L. Y., Engler, A. C., Hedrick, J. L. \& Yang, Y. Y. Antibacterial and antifouling catheter coatings using surface grafted PEG-b-cationic polycarbonate diblock copolymers. Biomaterials 33, 6593-6603 (2012).

18 Bass, M. \& Freger, V. Facile evaluation of coating thickness on membranes using ATR-FTIR. J. Membr. Sci. 492, 348-354 (2015).

19 Shi, C., Yuan, W., Khan, M., Li, Q., Feng, Y., Yao, F. \& Zhang, W. Hydrophilic PCU scaffolds prepared by grafting PEGMA and immobilizing gelatin to enhance cell adhesion and proliferation. Mater. Sci. Eng. C 50, 201-209 (2015).

20 Zhang, M., Li, X. H., Gong, Y. D., Zhao, N. M. \& Zhang, X. F. Properties and biocompatibility of chitosan films modified by blending with PEG. Biomaterials 23, 2641-2648 (2010).

21 Zhang, C., Bai, Y., Sun, Y., Gu, J. \& Xu, Y. Preparation of hydrophilic HDPE porous membranes via thermally induced phase separation by blending of amphiphilic PE-b-PEG copolymer. J. Membr. Sci. 365, 216-224 (2010).

22 Andreopoulos, F. M., Beckman, E. J. \& Russell, A. J. Light-induced tailoring of PEG-hydrogel properties. Biomaterials 19, 1343-1352 (1998).
23 Iza, M., Stoianovici, G., Viora, L., Grossiord, J. L. \& Couarraze, G. Hydrogels of poly(ethylene glycol): mechanical characterization and release of a model drug. J. Controlled Release 52, 41-51 (1998).

24 Cruise, G. M., Scharp, D. S. \& Hubbell, J. A. Characterization of permeability and network structure of interfacially photopolymerized poly(ethylene glycol) diacrylate hydrogels. Biomaterials 19, 1287-1294 (1998).

$25 \mathrm{Kim}$, I. S., Jeong, Y. I. \& Kim, S. H. Self-assembled hydrogel nanoparticles composed of dextran and poly(ethylene glycol) macromer. Int. J. Pharm. 205, 109-116 (2000).

26 Lee, S. J., Kim, S. S. \& Lee, Y. M. Interpenetrating polymer network hydrogels based on poly(ethylene glycol) macromer and chitosan. Carbohydr. Polym. 41, 197-205 (2000).

27 Bhattarai, N., Ramay, H. R., Gunn, J., Matsen, F. A. \& Zhang, M. PEG-grafted chitosan as an injectable thermosensitive hydrogel for sustained protein release. J. Controlled Release 103, 609-624 (2005).

28 Ajji, Z., Othman, I. \& Rosiak, J. M. Production of hydrogel wound dressings using gamma radiation. Nucl. Instrum. Methods Phys. Res. Sect. B 229, 375-380 (2005).

$29 \mathrm{Fu}$, H., Rahaman, M. N., Brown, R. F. \& Day, D. E. Evaluation of BSA protein release from hollow hydroxyapatite microspheres into PEG hydrogel. Mater. Sci. Eng. C 33, 2245-2250 (2013).

30 Ito, T., Yoshida, C. \& Murakami, Y. Design of novel sheet-shaped chitosan hydrogel for wound healing: a hybrid biomaterial consisting of both PEG-grafted chitosan and crosslinkable polymeric micelles acting as drug containers. Mater. Sci. Eng. C 33, 3697-3703 (2013).

31 Lin, N. J., Yang, H. S., Chang, Y., Tung, K. L., Chen, W. H., Cheng, H. W., Hsiao, S. W., Aimar, P., Yamamoto, K. \& Lai, J. Y. Surface self-assembled PEGylation of fluoro-Based PVDF membranes via hydrophobic-driven copolymer anchoring for ultra-stable biofouling resistance. Langmuir 29, 10183-10193 (2013).

32 Schroeder, M. E., Zurick, K. M., McGrath, D. E. \& Bernards, M. T. Multifunctional polyampholyte hydrogels with fouling resistance and protein conjugation capacity. Biomacromolecules 14, 3112-3122 (2013).

33 Gunkel, G. \& Huck, W. T. S. Cooperative adsorption of lipoprotein phospholipids, triglycerides, and cholesteryl esters are a key factor in nonspecific adsorption from blood plasma to antifouling polymer surfaces. J. Am. Chem. Soc. 135, 7047-7052 (2013).

34 Yuan, W., Feng, Y. Wang, H., Yang, D., An, B., Zhang, W., Khan, M. \& Guo, J. Hemocompatible surface of electrospun nanofibrous scaffolds by ATRP modification. Mater. Sci. Eng. C 33, 3644-3651 (2013).

35 Abednejad, A. S., Amoabediny, G. \& Ghaee, A. Surface modification of polypropylene membrane by polyethylene glycol graft polymerization. Mater. Sci. Eng. C 42, 443-450 (2014).

36 Higuchi, A., Sugiyama, K., Yoon, B. O., Sakurai, M., Hara, M., Sumita, M., Sugawara, S. I. \& Shirai, T. Serum protein adsorption and platelet adhesion on pluronic $^{\mathrm{TM}}$-adsorbed polysulfone membranes. Biomaterials 24, 3235-3245 (2003).

37 Tsai, W. B., Grunkemeier, J. M. \& Horbett, T. A. Human plasma fibrinogen adsorption and platelet adhesion to polystyrene. J. Biomed. Mater. Res. 44, 130-139 (1999).

38 Grunkemeier, J. M., Tsai, W. B., Alexander, M. R., Castiner, D. G. \& Horbett, T. A. Platelet adhesion and procoagulant activity induced by contact with radiofrequency glow discharge polymers: roles of adsorbed fibrinogen and vWF. J. Biomed. Mater. Res. 51, 669-679 (2000). 